\title{
Connection of Nicotine to Diet-Induced Obesity and Non-Alcoholic Fatty Liver Disease: Cellular and Mechanistic Insights
}

\author{
Amiya P. Sinha-Hikim ${ }^{1,2 *}$, Indrani Sinha-Hikim ${ }^{1,2}$ and Theodore C. Friedman ${ }^{1,2}$ \\ ${ }^{1}$ Division of Endocrinology, Metabolism and Molecular Medicine, Department of Internal Medicine, Charles R. Drew \\ University of Medicine and Science, Los Angeles, CA, USA, ${ }^{2}$ David Geffen School of Medicine at University of California, \\ Los Angeles, CA, USA
}

OPEN ACCESS

Edited by:

Toru Hosoi,

Hiroshima University, Japan

Reviewed by:

Maristela Oliveira Poletini, Universidade Federal de Minas

Gerais, Brazil

Xuanzhi Zhan,

Vanderbilt University, USA

${ }^{*}$ Correspondence:

Amiya P. Sinha-Hikim amiyasinhahikim@cdrewu.edu

Specialty section: This article was submitted to Cellular Endocrinology, a section of the journal

Frontiers in Endocrinology

Received: 24 October 2016 Accepted: 25 January 2017 Published: 10 February 2017

Citation: Sinha-Hikim AP, Sinha-Hikim I and Friedman TC (2017) Connection of Nicotine to Diet-Induced Obesity and Non-Alcoholic Fatty Liver Disease: Cellular and Mechanistic Insights.

Front. Endocrinol. 8:23. doi: 10.3389/fendo.2017.00023
Non-alcoholic fatty liver disease (NAFLD) poses a serious health hazard affecting 20-40\% of adults in the general population in the USA and over $70 \%$ of the obese and extremely obese people. In addition to obesity, nicotine is recognized as a risk factor for NAFLD, and it has been reported that nicotine can exaggerate obesity-induced hepatic steatosis. The development of NAFLD has serious clinical complications because of its potential progression from simple hepatic steatosis to non-alcoholic steatohepatitis (NASH), liver cirrhosis, and hepatocellular carcinoma. Multiple mechanisms can be involved in nicotine plus high-fat diet-induced (HFD) hepatic steatosis. Emerging evidence now suggests that nicotine exacerbates hepatic steatosis triggered by HFD, through increased oxidative stress and hepatocellular apoptosis, decreased phosphorylation (inactivation) of adenosine-5-monophosphate-activated protein kinase and, in turn, up-regulation of sterol response-element binding protein 1-c, fatty acid synthase, and activation of acetyl-coenzyme A-carboxylase, leading to increased hepatic lipogenesis. There is also growing evidence that chronic endoplasmic reticulum stress through regulation of several pathways leading to oxidative stress, inflammation, perturbed hepatic lipid homeostasis, apoptosis, and autophagy can induce hepatic steatosis and its progression to NASH. Evidence also suggests a central role of the gut microbiota in obesity and its related disorders, including NAFLD. This review explores the contribution of nicotine and obesity to the development of NAFLD and its molecular underpinning.

Keywords: nicotine, high-fat diet, obesity, oxidative stress, non-alcoholic fatty liver disease

\section{INTRODUCTION}

In 2009, approximately $20 \%$ ( 60 million) of Americans smoked and about $\sim 88$ million non-smokers were exposed to secondhand smoke (1). Unless dramatic progress is made in diminishing the initiation and increasing cessation of combustible tobacco product use, a billion of preventable death will occur in twenty-first century worldwide (2). Thus cigarette smoking needs to be viewed as a chronic disease, and in addition to research on the difficult problem of smoking cessation, research also needs to be conducted on the detrimental effects of chronic cigarette use. The prevalence of smoking was $31.1 \%$ among persons below the federal poverty level (1), so smoking should be considered a health disparity. Cigarette smoking is the leading preventable cause of death and disability worldwide $(3,4)$. Smoking is a major risk factor for chronic obstructive pulmonary disease and lung cancer and devastating cardiovascular disease (CVD), such as myocardial infarction, sudden death, stroke, 
and peripheral vascular disease (5-8), with a dose-response correlation between CVD morbidity and mortality and the number of cigarettes smoked (8). Furthermore, usages of nicotine only formulations, such as transdermal patches, nicotine gum, and electronic cigarettes, in particular, are increasing $(9,10)$. The lack of targeted and effective strategies to control tobacco consumption contribute to large burden of cardiovascular disorders in low- and middle-income people worldwide, where CVD has become the leading cause of morbidity and mortality (8). Moreover, smoking leads to substantial financial costs to society. Between 2009 and 2012, smoking cost the USA approximately \$289-332.5 billion, with $46-53 \%$ of this amount spent on adult medical care and the rest due to loss of workplace productivity (4). The negative effects of smoking, thus, leads to reduced quality of life and loss of life and can lead to personal and national financial burden. The health risk associated with smoking can be exaggerated by obesity $(11,12)$.

Nicotinic acetylcholine receptors (nAChRs) are a family of ionotropic receptor proteins formed by five homologous or identical subunits and are involved in signal transduction between neurons and muscle cells $(10,13,14)$. nAChRs are divided into muscle $(\alpha 1, \beta 1, \gamma / \varepsilon$, and $\delta$ ) and neuronal nAChRs ( $\alpha 2-10$ and $\beta$ 2-4) $(10,14,15)$. Neuronal nAChRs are further subdivided into those that form homomeric receptors when expressed in heterologous systems $(\alpha 7-10)$ and those that form heteromeric receptors ( $\alpha 2-6$ and $\beta 2-4)$ in different combinations (10, 14, 15). nAChRs are also expressed in various tissues, including adipocytes, pancreatic beta cells, hepatocytes, myocytes, and cardiomyocytes (16-19). The nAChRs, which are activated by nicotine or its metabolites cotinine, can activate various signaling pathways that can alter cellular metabolic homeostasis (10). This review discusses emerging evidence of contribution of nicotine when combined with obesity to the development of hepatic steatosis and insights into the molecular mechanisms by which nicotine contributes to non-alcoholic fatty liver disease (NAFLD).

\section{NAFLD Is Highly Prevalent in Obese Individuals and Can Be Exaggerated by Smoking}

Non-alcoholic fatty liver disease is the most common liver disorder and is associated with metabolic syndrome and diabetes mellitus. It includes the whole spectrum of fatty liver, ranging from simple steatosis to steatohepatitis [non-alcoholic steatohepatitis (NASH)], which can progress to liver cirrhosis and hepatocellular carcinoma (20-22). Data from the Framingham Heart Study showed that fatty liver is characterized by dysglycemia and dyslipidemia independent of visceral adipose tissue (23). There is increasing evidence that smoking can also contribute to NAFLD. Multiple logistic regression analysis from a retrospective follow-up study over a 10-year period, involving 2,029 Japanese subjects, demonstrated that cigarette smoking (adjusted odd ratio 1.91; 95\% confidence interval 1.34-2.72) is an independent risk factor for NAFLD (24). A statistically significant association between smoking history and severity of liver fibrosis was demonstrated in a large multicenter cohort of 1,091 subjects with biopsy-proven NAFLD (25). Of further importance, the health risk associated with smoking, whether passive or active, is exaggerated by obesity, and smoking and obesity are the leading causes of morbidity and mortality worldwide (11, 12). The life expectancy of an obese smoker is 13 years less than that of a normal-weight non-smoker (11). Furthermore, smoking lowers the body weight and body mass index (BMI), which make many people reluctant to quit smoking (11).

In the United States, $72 \%$ of the adult male population is overweight or obese out of which $11 \%$ have a BMI of $35 \mathrm{~kg} / \mathrm{m}^{2}$ and $4 \%$ a BMI of at least $40 \mathrm{~kg} / \mathrm{m}^{2}(26)$. Obese men are at a higher risk to develop atherosclerosis, coronary heart disease, diabetes, hypertension, dyslipidemia, and NAFLD (27). NAFLD, in turn, can also be an independent risk factor of atherosclerosis and CVD $(28,29)$. Currently, $34 \%$ of the general population and over $75 \%$ of the obese and extremely obese individuals are estimated to have hepatic steatosis (30). Hispanics have the highest prevalence of hepatic steatosis followed by Caucasians and then AfricanAmericans (31).

\section{Mechanisms Linking Nicotine to NAFLD}

The hallmark of NAFLD is accumulation of triglycerides (TG) in the hepatocytes (steatosis). Multiple mechanisms have proposed to explain the accumulation of TG in the liver, including (i) increased dietary fat intake, (ii) excess free fatty acid (FFA) delivery from lipolysis of white adipose tissue, (iii) increased de novo lipogenesis, (iv) reduced fatty acid $\beta$-oxidation, and (v) reduced fat export in the form of very low-density lipoprotein (VLDL) $(21,32)$. The precise molecular mechanisms of the pathogenesis of steatosis and its progression to NASH are not well understood. AMP-activated protein kinase (AMPK) is a central regulator of lipid homeostasis and mediates suppression of lipogenic gene expression, such as acetyl-coenzyme A-carboxylase (ACC) and fatty acid synthase (FAS) through inhibition of sterol regulatory element binding protein-1c (SREBP1-c) and carbohydrate response-element binding protein (ChREBP) (33-35). ACC is the rate determining enzyme for the synthesis of malonyl-CoA, both a critical substrate for fatty acid biosynthesis and a potent inhibitor of fatty acid oxidation (33). AMPK can phosphorylate and inactivate ACC leading to inhibition of de novo fatty acid and cholesterol synthesis (33). AMPK can also increase the activity of malonyl-CoA decarboxylase to further decrease malonyl-CoA levels (33). Lipogenesis is further regulated by glucose, which activates ChREBP, which, in turn, activates gene expression of most enzymes involved in lipogenesis (21).

\section{Two-Hit or Multiple-Hit Hypothesis}

Steatosis can prime the liver to develop more progressive liver pathologies in response to additional metabolic and/or environmental stressors. Mechanistically, this is commonly mediated by the prevalent "two-hit" hypothesis that implies accumulation of TG in hepatocytes (steatosis) in the first hit, followed by triggering progression to inflammation, oxidative stress, and apoptosis in the second hit $(22,35,36)$. In more advanced cases, fibrosis is also exacerbated, leading to the progressive form of NAFLD, known as NASH. Environmental stressors [such as high-fat diet (HFD), cigarette smoke, drugs, and pollutants] or metabolic stressors (such as obesity, diabetes, hypertension, hypertriglyceridemia and hypercholesterolemia) are known to trigger progression to the second phase. Nonetheless, the molecular underpinning of 
steatosis is not well understood. Oxidative stress coupled with hepatocyte apoptosis is believed to play a pivotal role in pathogenesis of NAFLD $(22,37,38)$. In fact, emerging data suggest that hepatocyte apoptosis plays a key component in the progression of simple steatosis to NASH $(22,37)$. Notably, a proof-of-principle, randomized, double blind, placebo-controlled study of GS-9450 (selective inhibitor of caspases 1,8, and 9) suggests that reducing hepatocellular apoptosis may be a valuable therapeutic strategy in patients with NASH (39).

\section{Smoking Exacerbates Effects of Dietary Fat on Liver}

Animal experiments using first-hand (delivered via a smoking device designed to puff the smoke into the inhalation chamber housing the animals), second-hand smoke (side-stream whole smoke solution delivered via a puffer box), or nicotine and models of genetic or diet-induced obesity (DIO) provide perhaps the strongest evidence linking nicotine to hepatic steatosis and NAFLD. Yuan and colleagues (40) demonstrated that HFD-fed apoB100 transgenic mice on C57Bl6J background exposed to second-hand smoke exhibit lipid accumulation in the liver and this effect was mediated by inactivation of AMPK and activation of its downstream target SREBP-1. In another study, Azzalini and colleagues (41) demonstrated that first-hand smoke exacerbates NAFLD in obese Zucker rats. The effect of first-hand smoke on the severity of hepatic steatosis was associated with increased oxidative stress, hepatocyte apoptosis, expression of key genes involved in hepatic fibrogenesis, and inactivation of Akt but stimulation of extracellular signal regulated kinase (ERK) signaling. We used the model of DIO in C57BL6J mice to study the mechanisms underlying the detrimental effects of nicotine and HFD in the development of fatty liver disease (42). Like humans, these mice, when fed a HFD deriving $60 \%$ of calories from fat, developed visceral adiposity, hyperglycemia, insulin and leptin resistance, as well as hepatic steatosis $(43,44)$. We elected to use a single drug (nicotine) as opposed to first-or second-hand smoke in order to eliminate the confounding effects of other components involved in cigarette smoking. Adult C57BL6 male mice were fed a normal chow diet or HFD and received twice daily injections of nicotine $(0.75 \mathrm{mg} / \mathrm{kg} \mathrm{BW}, \mathrm{IP})$ or saline for 10 weeks. Of note, the daily dosage of $1.5 \mathrm{mg} / \mathrm{kg}$ BW in mice results in a serum concentration of nicotine that is similar to the clinically relevant concentrations found in habitual cigarette smokers and nicotine-containing chewing gum users (19). We purposely used shorter (10-week) duration to examine the synergistic effects of these two insults in the initiation of NAFLD, as a longer exposure to HFD alone results in extensive steatosis (45) and systemic inflammation (46). We found that nicotine alone did not lead to hepatic steatosis, but it caused hepatic steatosis only when combined with HFD (Figure 1) (42). A significant $(p<0.01)$ increase in the $\mathrm{Vv} \%$ of lipid droplets together with a reduction in the $\mathrm{Vv} \%$ of endoplasmic reticulum (ER) (67.8\%) and glycogen (49.2\%) was also noted in hepatocytes from mice on HFD plus nicotine, compared to mice on HFD alone. The additive effects of nicotine on the severity of HFD-induced hepatic steatosis was associated with significantly greater oxidative stress, increased hepatic TG levels, higher incidence of hepatocellular apoptosis, inactivation (dephosphorylation) of AMPK, and activation of its downstream target ACC (42).

Indeed, these above studies, using various experimental models, demonstrated that nicotine further worsens HFD-induced hepatic steatosis. Summation of the results further indicate that increased oxidative stress and hepatocellular apoptosis, inactivation of Akt and AMPK, and activation of its downstream targets SREBP-1 and ACC, together with stimulation of ERK are involved in the pathogenesis of nicotine plus HFD-induced hepatic steatosis.

\section{Nicotine exacerbates HFD-induced hepatic steatosis in obese mice}
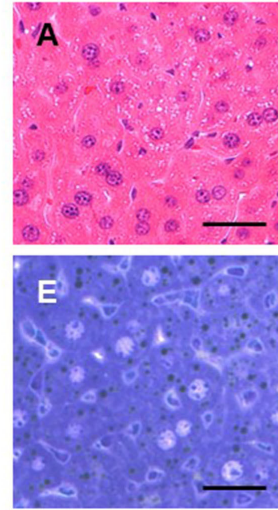

NCD+SAL
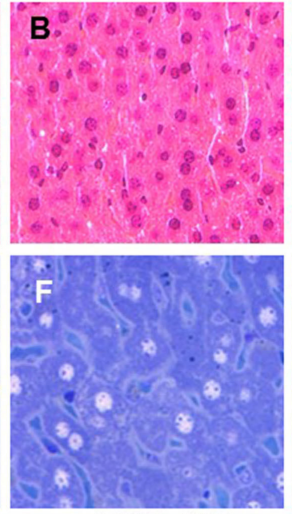

$\mathrm{NCD}+\mathrm{NIC}$

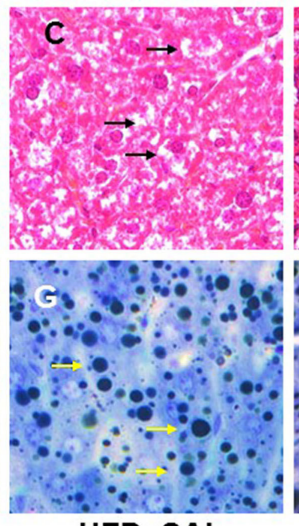

HFD+SAL

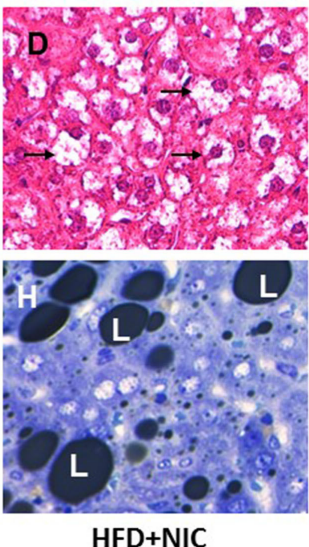

HFD+NIC

FIGURE 1 | Representative H\&E-stained liver sections from mice fed with normal chow diet (NCD) without (A) or with (B) nicotine exhibit normal histological appearance. Compared with a mouse on a high-fat diet (HFD), where a modest increase in lipid accumulation (arrow) is detected (C), combined treatment with nicotine and HFD causes a marked increase in lipid accumulation in the liver (D). (E-H) Representative light microscopic images of glutaraldehydefixed, osmium tetroxide post-fixed, epoxy-embedded, and toluidine-blue-stained live sections from different treatment groups show nicotine plus a HFD (H) causes a striking increase in lipid accumulation of varying sizes in hepatocytes compared to those from mice on a HFD alone [(G), arrow]. Mice fed with NCD with (F) or without nicotine $(\mathbf{E})$ have normal liver morphology. Scale bar $=25 \mu \mathrm{m}$ [reproduced with permission from Friedman et al. (42)]. 


\section{Contribution of Adipose Tissue Lipolysis to Nicotine and HFD-Induced Hepatic Steatosis}

Adipose tissue has the unique function of storing TG in lipid droplets and upon lipolysis, to provide FFA to other organs during time of energy shortage (47). In obesity and other conditions where cellular lipid homeostasis is perturbed, lipolysis can contribute to ectopic lipid accumulation (48). Mounting experimental evidence supports that nicotine considerably decreases HFD-induced adiposity in mice, as determined by dual-energy $\mathrm{X}$-ray absorption densitometry, computed tomography, as well as by magnetic resonance imaging, with no change in lean body mass $(19,49)$. Nicotine when combined with a HFD, however, significantly increases the levels of serum, hepatic TG, and circulating FFA $(19,42,50)$. These results indicate that nicotine in mice on a HFD promotes lipid distribution from adipose tissue to other organs. Decisive evidence that increased adipose tissue lipolysis contributes to nicotine plus HFD-induced hepatic steatosis derives from studies showing that acipimox, an inhibitor of adipose tissue lipolysis, treatment significantly prevented nicotine plus HFD-induced increase in hepatic TG levels and hepatic steatosis (Figure 2) (42). A recent study (19) has also demonstrated that acipimox treatment significantly prevented nicotine plus HFD-induced increase in serum FFA levels and serum and hepatic TG levels, as well as hepatic steatosis (Figure 2). This concept is supported by another evidence showing that inhibition of adipose tissue lipolysis by adipose-specific ablation of desnutrin prevented ectopic lipid accumulation in the liver even when fed with a HFD (51). Together, these results suggest that adipose tissue lipolysis plays a major role in the development of nicotine plus HFD-induced hepatic steatosis.

Mechanistically, nicotine activates AMPKo2 in adipocytes, which phosphorylates MAP kinase phosphatase-1 (MKP1) at serine 334, resulting its proteasome-dependent degradation (19). Nicotine-induced reduction in MKP1, in turn, activates both p38 mitogen-activated protein kinase (p38 MAPK) and c-jun- $\mathrm{NH}_{2}-$ terminal kinase (JNK), which phosphorylates insulin receptor substrate 1 (IRS1) at serine 307. Phosphorylation of IRS1 leads to its degradation and the subsequent inhibition of Akt, resulting in increased adipose tissue lipolysis and circulating FFA levels (19).

\section{The Role of ER Stress}

Chronic ER stress induces several pathways leading to oxidative stress, inflammation, perturbed hepatic lipid homeostasis, apoptosis, and autophagy that can lead to hepatic steatosis and its progression to NASH [reviewed in Ref. (52)]. ER stress is related with hepatic lipid metabolism by directly increasing lipogenesis and limiting VLDL formation. It has been demonstrated that

\section{Potential mechanisms linking nicotine to NAFLD in obese mice}

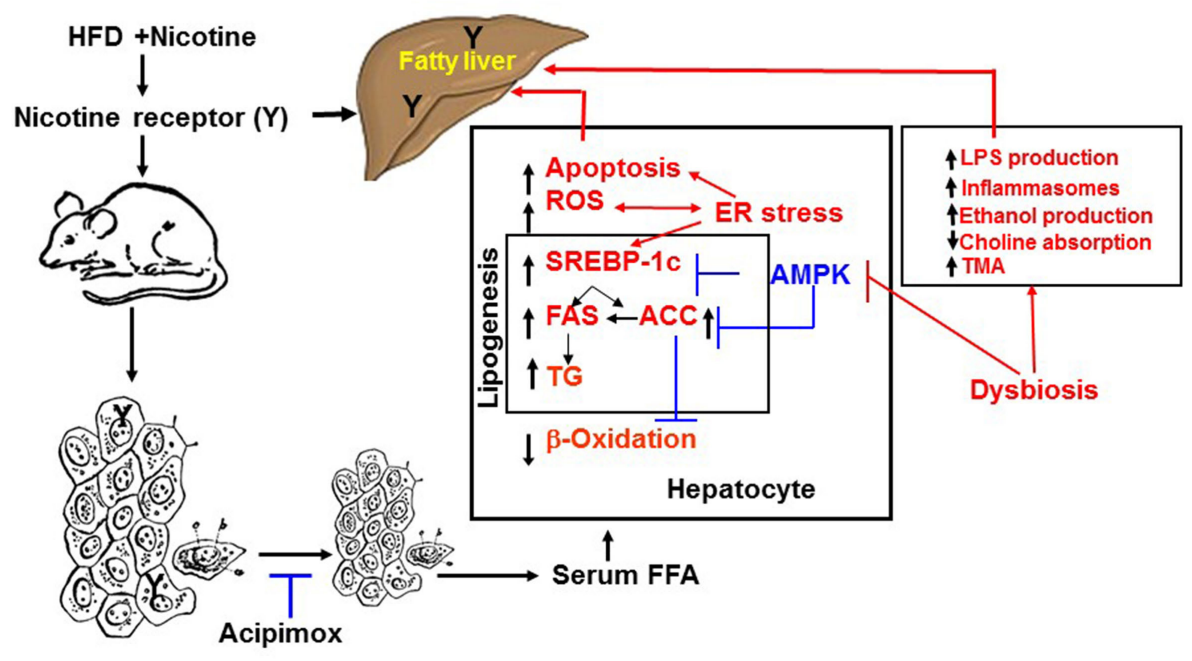

FIGURE 2 | Potential mechanisms of nicotine plus HFD-induced hepatic steatosis in obese mice. Nicotine plus a HFD promotes abdominal lipolysis, resulting in free fatty acid (FAA) release from adipose tissue into the circulation, thereby contributing to the buildup of lipids as triglyceride in the liver. In addition, nicotine plus a HFD may also promote de novo lipogenesis through inactivation of AMP-activated protein kinase (AMPK) and activation of its downstream target acetyl-coenzyme A-carboxylase (ACC), leading to the development of hepatic steatosis. Inactivation of AMPK can also stimulate lipogenesis through upregulation of key genes in the lipogenic pathway, such as fatty acid synthase (FAS) and ACC, by activating the transcription factor sterol regulatory element binding protein $1 \mathrm{c}$ (SREBP-1C). Intrahepatic lipid accumulation can also trigger hepatocellular apoptosis through generation of oxidative stress coupled with activation of c-Jun $\mathrm{NH}_{2}$-termina kinase (JNK)-mediated apoptotic signaling. AMPK inactivation could further sensitize liver cells to nicotine plus HFD-induced apoptosis. There is also growing evidence that chronic endoplasmic reticulum stress through regulation of several pathways leading to oxidative stress, inflammation, perturbed hepatic lipid homeostasis, apoptosis, and autophagy, can also induce hepatic steatosis and its progression to non-alcoholic steatohepatitis. Evidence also suggests a central role of the gut microbiota in obesity and its related disorders, including non-alcoholic fatty liver disease (NAFLD). It is possible nicotine plus a HFD through changes in short-chain fatty acids metabolism, increased intestinal permeability and lipopolysaccharides activation of Toll-like receptors and inflammasomes, endogenous ethanol production, decreased choline availability and increased trimethylamine (TAM) production could cause NAFLD. The multiple mechanisms of nicotine and obesity-induced hepatic steatosis can results from both its nicotinic acetylcholine receptor-mediated and non-receptor effects. 
ER stress contributes to increased hepatic lipogenesis in $o b / o b$ mice through SREBP1c activation while overexpression of ER chaperone BIP decreased ER stress and inhibited lipogenesis by inactivating SREBP1 (53). Furthermore, ER stress modulates several factors, including nuclear factor 2 erythroid-related factor 2 (Nrf2), JNK, nuclear factor $\kappa \mathrm{B}(\mathrm{NF}-\kappa \mathrm{B})$, and c/EBP homologous protein $(\mathrm{CHOP})$, all of which play a role in the inflammatory process, cellular defense against oxidative stress, and cell death. For example, Nrf2 serves as master regular of a cellular defense system against oxidative stress $(54,55)$. Under physiological conditions, $\mathrm{Nrf} 2$ is sequestered in the cytoplasm by Keap1, which facilitates its ubiquitination and proteasomic degradation. Upon exposure to oxidative stress, the sequestration complex brakes down and the dissociated Nrf2 translocates into the nucleus, where it binds to cisacting antioxidant response elements and promotes the transcription of a large number of cytoprotective genes $(56,57)$. However, under pathological conditions, such as NASH, NRf2 activity is impaired (52). Consistent with the role of NrF2 in NAFLD, it has been demonstrated that genetic ablation of Nrf2 markedly exacerbates NASH (58). Conversely, enhanced expression of Nrf2 in mice bearing a hepatocyte-specific knockdown of Keap1 attenuated the fatty liver induced by a methionine- and choline-deficient diet (59). JNK is activated in various animal models of obesity and also in patients with NASH and its deletion results in attenuation of fatty liver (22). Activation of JNK has also been documented in HFD-induced hepatic steatosis in apoplipoprotein E knockout mice (60) or nicotine plus HFD-induced hepatic steatosis in obese mice (42). NF- $\mathrm{KB}$ is a transcription factor and a primary regulator of inflammatory action. Activation of NF- $\kappa B$ dimers is due to inhibiton of NF- $\mathrm{KB}$ kinase (IKK)-mediated phosphorylationinduced proteasomal degradation of I $\kappa$, enabling the active $\mathrm{NF}-\kappa \mathrm{B}$ transcription factor subunits to translocate to the nucleus and induce target gene expression. Persistent activation of NF- $\mathrm{KB}$ signaling has been shown in animal models of NAFLD as well as in patients with NASH (35). Furthermore, CHOP plays a pivotal role in ER-induced cell death. Deletion of CHOP decreases hepatocyte apoptosis in alcohol-induced liver disease and reduces cholestsis-induced liver fibrosis $(61,62)$.

It is worth noting here that both nicotine $(63,64)$ and HFD $(65,66)$ are capable of generating hepatic ER stress. Thus, it is possible that nicotine plus HFD could generate severe hepatic ER stress leading to hepatic steatosis. Clearly, further studies are needed to define the role of ER stress in fatty liver disease triggered by nicotine and HFD.

\section{Connections of Gut Microbiota to NAFLD}

Evidence linking dysbiosis (also known as disruption of the normal gut microbiota) contributes to the pathogenesis of NAFLD has accumulated rapidly (67-69). Early studies have shown that patients with biopsy-proven NAFLD had significantly increased gut permeability compared to healthy volunteers (70). Both the increased gut permeability and prevalence of small intestinal bacterial overgrowth correlated with severity of steatosis in the patients with the NAFHD (70). The strongest evidence supporting the role of dysbiosis in NAFLD, however, stems from animals studies where the gut microbiome has been manipulated. It has been shown that microbiome from obese mice is linked to increased energy from the diet and this trail can be transmissible to lean adult germ-free mice by co-housing with obese mice (71). A growing number of studies examining how dysbiosis might drive NAFLD have identified a number of plausible mechanisms, including changes in short-chain fatty acids (SCFAs) metabolism, increased intestinal permeability and lipopolysaccharides (LPS) activation of toll-like receptors (TLRs) and inflammasomes, endogenous ethanol production, decreased choline availability, and trimethylamine production (69). For example, it has been shown that SCFAs can lower FAS activity and hepatic lipid synthesis in HFD-fed mice through activation of AMPK and inactivation of its downstream substrate ACC (72). Evidence exists that smoking can also induce profound changes in intestinal microbiota $(73,74)$. Taken together, it is possible that nicotine plus a HFD through changes in SCFAs metabolism, increased intestinal permeability and LPS activation of TLRs and inflammasomes, endogenous ethanol production, decreased choline availability and trimethylamine production could cause NAFLD.

\section{CONCLUSION AND PERSPECTIVES}

Nicotine when combined with a HFD leads to NAFLD through multiple mechanisms, summarized in Figure 2, including generation of severe oxidative stress and increased hepatocellular apoptosis as well inducing adipose tissue lipolysis resulting in excess delivery of FFA and perturbation of hepatic lipid homeostasis through inactivation of AMPK. There is also growing evidence that chronic ER stress through regulation of several pathways leading to oxidative stress, inflammation, perturbed hepatic lipid homeostasis, apoptosis, and autophagy, can also induce hepatic steatosis and its progression to NASH. Evidence also suggests a central role of the gut microbiota in obesity and its related disorders, including NAFLD. The multiple mechanisms of nicotine and obesity-induced hepatic steatosis is mediated by both its nAChR-mediated and non-receptor effects.

A better understanding of the mechanisms and various diverse signaling pathways responsible for nicotine plus HFD-induced NAFLD may also unveil novel pharmacological targets to treat fatty liver disease and adverse metabolic sequelae. The emerging knowledge about a direct connection of smoking or tobacco products to obesity and fatty liver disease should be considered during the evaluation of regulations on nicotine product manufacturing, distribution, and marketing.

\section{AUTHOR CONTRIBUTIONS}

AS-H and IS-H conceived and prepared the manuscript. TF critically appraised the manuscript and also wrote a part of the manuscript.

\section{FUNDING}

This work was supported by the Diversity Promoting Institution Drug Abuse Research Program (DIDARP) grant (R24DA017298) and the Accelerating Excellence in Translational Science (AXIS) grant (2U54MD007598) from the National Institutes of Health (NIH). 


\section{REFERENCES}

1. CDC. Vital signs: nonsmokers' exposure to secondhand smoke - United States, 1999-2008. MMWR Morb Mortal Wkly Rep (2010) 59:1141-6.

2. McAfee TA, Krugar J. Tobacco use patterns. J Environ Public Health (2012) 2012:564930. doi:10.1155/2012/564390

3. He J, Gu D, Wu X, Reynolds K, Duan X, Yao C, et al. Major causes of death among men and women in China. N Engl J Med (2005) 353:1124-34. doi:10.1056/NEJMsa050467

4. Harris KK, Zopey M, Friedman TC. Metabolic effects of smoking cessation. Nat Rev Endocrinol (2016) 12:299-308. doi:10.1038/nrendo.2016.32

5. Barnes PJ. New concepts in chronic obstructive pulmonary disease. Ann Rev Med (2003) 75:19-37.

6. Hudson NL, Mannino DM. Tobacco use: a chronic illness? J Community Health (2010) 35:549-53. doi:10.1007/s10900-010-9241-x

7. Zaher C, Halbert R, Dubios R, George D, Nonikov D. Smoking-related diseases: the importance of COPD. Int J Tuberc Lung (2004) 8:1423-8.

8. Saleheen D, Zhao W, Rasheed A. Epidemiology and public health policy of tobacco use and cardiovascular disorders in low-and middle-income countries. Arterioscler Tromb Vasc Biol (2014) 34:1811-9. doi:10.1161/ ATVBAHA.114.303826

9. Colombo ES, Davis J, Makvandi M, Aragon M, Lucas SN, Paffett ML, et al. Effects of nicotine on cardiovascular remodeling in a mouse model of systemic hypertension. Cardiovasc Toxicol (2013) 13:364-9. doi:10.1007/ s12012-013-9217-z

10. Grando SA. Connections of nicotine to cancer. Nat Rev Cancer (2014) 14:419-29. doi:10.1038/nrc3725

11. Chiolero A, Faeh D, Paccaud F, Cornuz J. Consequences of smoking for body weight, body fat distribution, and insulin resistance. Am J Clin Nutr (2008) 87:801-9.

12. Haslam DW, James WP. Obesity. Lancet (2005) 366:1197-209. doi:10.1016/ S0140-6736(05)67483-1

13. Albuquerque EX, Alkondon M, Pereira EF, Castro NG, Schrattenholz A, Barbosa CT, et al. Properties of neuronal nicotinic acetylcholine receptors: pharmacological characterization and modulation of synaptic function. J Pharmacol Exp Ther (1997) 280:1117-36.

14. Lindstrom JM. Nicotinic acetylcholine receptors of muscles and nerves: comparison of their structures, functional roles, and vulnerability to pathology. Ann N Y Acad Sci (2003) 998:41-52. doi:10.1196/annals.1254.007

15. Gottic C, Carbonnelle E, Moretti M, Zwart R, Clementi F. Drugs selective for nicotinic receptors subtypes: a real possibility or a dream? Behav Brain Res (2000) 113:183-92. doi:10.1016/S0166-4328(00)00212-6

16. Liu RH, Mizuta M, Matsukura S. The expression and functional role of nicotinic acetylcholine receptors in rat adipocytes. J Pharmacol Exp Ther (2004) 310:52-8. doi:10.1124/jpet.103.065037

17. Jutkiewicz EM, Rice KC, Carroll FI, Woods JH. Patterns of nicotinic receptor antagonism II. Cardiovascular effects in rats. Drugs Alcohol Depen (2013) 131:284-97. doi:10.1016/j.drugalcdep.2012.12.021

18. Vu CU, Siddiqui JA, Wadensweiler P, Gayen JR, Avolio E, Bandyopadhyay GK, et al. Nicotinic acetylcholine receptors in glucose homeostasis: the acute hyperglycemic and chronic insulin-sensitive effects of nicotine suggests dual opposing roles of the receptors in male mice. Endocrinology (2014) 155:3793-5.

19. Wu Y, Song P, Zhang W, Liu J, Dai X, Liu Z, et al. Activation of AMPK $\alpha 2$ in adipocytes is essential for nicotine-induced insulin resistance in vivo. Nat Med (2015) 21:373-82. doi:10.1038/nm.3826

20. Farrell GC, Teoh NC, McCuskey RS. Hepatic microcirculation in fatty liver disease. Anat Rec (2008) 291:684-92. doi:10.1002/ar.20715

21. Postic C, Girard J. Contribution of de novo fatty acid synthesis to hepatic steatosis and insulin resistance: lessons from genetically engineered mice. J Clin Invest (2008) 118:829-38. doi:10.1172/JCI34275

22. Trauner M, Arrease M, Wangner M. Fatty liver and lipotoxicity. Biochem Biophys Acta (2010) 1801:299-310. doi:10.1016/j.bbalip.2009.10.007

23. Speliotes EK, Massaro JM, Hoffmann U, Vasan RS, Meigs JB, Sahani DV, et al. Fatty liver is associated with dyslipidemia and dysglycemia independent of visceral fat: the Framingham Heart Study. Hepatology (2010) 51:1979-87. doi:10.1002/hep. 23593

24. Hamabe A, Uto H, Imamura Y, Kusano K, Mawatari S, Kumagai K, et al. Impact of cigarette smoking on onset of nonalcoholic fatty liver disease over a 10-year period. J Gastroenterol (2011) 46:769-78. doi:10.1007/s00535-0110376-z

25. Zein CO, Unalp A, Colvin R, Liu YC, McCullough AJ. Smoking and severity of hepatic fibrosis in nonalcoholic fatty liver disease. J Hepatol (2011) 54:753-9. doi:10.1016/j.jhep.2010.07.040

26. Friedman JM. Modern science versus the stigma of obesity. Nat Med (2004) 10:563-9. doi:10.1038/nm0604-563

27. Flegal KM, Carroll MD, Ogden CL, Curtin LR. Prevalence and trends in obesity among US adults, 1999-2008. JAMA (2010) 303:235-41. doi:10.1001/ jama.2009.2014

28. Targher G, Bertolini L, Poli F, Rodella S, Scala L, Tessari R, et al. Nonalcoholic fatty liver disease and risk of future cardiovascular events among type 2 diabetic patients. Diabetes (2005) 54:3541-6. doi:10.2337/diabetes.54.12.3541

29. Hamaguchi M, Kojima T, Takeda N, Nagata C, Takeda J, Sarui H, et al. Nonalcoholic fatty liver disease is a novel predictor of cardiovascular disease. World J Gastroenterol (2007) 13:1579-84. doi:10.3748/wjg.v13.i10.1579

30. Browning JD, Szczepaniak LS, Dobbins R, Nuremberg P, Horton JD, Cohen JC, et al. Prevalence of hepatic steatosis in an urban population in the United States: impact of ethnicity. Hepatology (2004) 40:1387-95. doi:10.1002/ hep. 20466

31. Browning JD, Horton JD. Molecular mediators of hepatic steatosis and liver injury. J Clin Invest (2004) 114:147-52. doi:10.1172/JCI200422422

32. Fabbrini E, Sullivan S, Klein S. Obesity and nonalcoholic fatty liver disease: biochemical, metabolic, and clinical implications. Hepatology (2010) 51:679-89. doi:10.1002/hep. 23280

33. Zhang BB, Zhou G, Li C. AMPK: an emerging drug target for diabetes and the metabolic syndrome. Cell Metab (2009) 9:407-16. doi:10.1016/ j.cmet.2009.03.012

34. Mihaylova MM, Shaw RJ. The AMPK signalling pathway coordinates cell growth, autophagy and metabolism. Nat Cell Biol (2011) 13:1016-23. doi:10.1038/ncb2329

35. Buzzetti E, Pinzani M, Tsochatzis EA. The multiple-hit pathogenesis of non-alcoholic fatty liver disease (NAFLD). Metabolism (2016) 65:1038-48. doi:10.1016/j.metabol.2015.12.012

36. Mantena S, King A, Andringa K, Eccleston H, Bailey S. Mitochondrial dysfunction and oxidative stress in the pathogenesis of alcohol- and obesity-induced fatty liver diseases. Free Radic Biol Med (2008) 44:1259-72. doi:10.1016/ j.freeradbiomed.2007.12.029

37. Kojima H, Sakurai S, Uemura M, Fukui H, Morimoto H, Tamagawa Y. Mitochondrial abnormality and oxidative stress in nonalcoholic steatohepatitis. Alcohol Clin Exp Res (2007) 31:S61-6. doi:10.1111/j.1530-0277.2006. 00288.x

38. Spahis S, Delvin E, Borys JM, Levy E. Oxidative stress as a critical factor in nonalcoholic fatty liver disease pathogenesis. Anioxid Redox Signal (2016). doi:10.1089/ars.2016.6776

39. Ratziu V, Sheikh MY, Sanyal AJ, Lim JK, Conjeevaram H, Chalasani N, et al. A phase 2, randomized, double-blind, placebo-controlled study of GS-9450 in subjects with nonalcoholic steatohepatitis. Hepatology (2012) 55:419-28. doi:10.1002/hep. 24747

40. Yuan H, Shyy JY, Martins-Green M. Second-hand smoke stimulates lipid accumulation in the liver by modulating AMPK and SREBP-1. J Hepatol (2009) 51:535-47. doi:10.1016/j.jhep.2009.03.026

41. Azzalini L, Ferrer E, Ramalho LN, Moreno M, Dominguez M, Colmenero J, et al. Cigarette smoking exacerbates nonalcoholic fatty liver disease in obese rats. Hepatology (2010) 51:1567-76. doi:10.1002/hep.23516

42. Friedman TC, Sinha-Hikim I, Parveen M, Najjar SM, Liu Y, Mangubat M, et al. Additive effects of nicotine and high-fat diet on hepatic steatosis in mice. Endocrinology (2012) 153:5809-20. doi:10.1210/en.2012-1750

43. Collins S, Martin TL, Surwit RS, Robidoux J. Genetic vulnerability to diet-induced obesity in the C57BL/6J mouse: physiological and molecular characteristics. Physiol Behav (2004) 81:243-8. doi:10.1016/j.physbeh.2004. 02.006

44. de Meijer VE, Le HD, Meisel JA, Akhavan Sharif MR, Pan A, Nose V, et al. Dietary fat intake promotes the development of hepatic steatosis independently from excess caloric consumption in a murine model. Metabolism (2010) 59:1092-105. doi:10.1016/j.metabol.2009.11.006

45. Zhang W, Kudo H, Kawai K, Fujisaka S, Usui I, Sugiyama T, et al. Tumor necrosis factor-alpha accelerates apoptosis of steatotic hepatocytes from 
a murine model of non-alcoholic fatty liver disease. Biochem Biophys Res Commun (2010) 391:1731-6. doi:10.1016/j.bbrc.2009.12.144

46. Kim F, Pham M, Maloney E, Rizzo NO, Morton GJ, Wisse BE, et al. Vascular inflammation, insulin resistance, and reduced nitric oxide production precede the onset of peripheral insulin resistance. Arterioscler Thromb Vasc Biol (2008) 28:1982-8. doi:10.1161/ATVBAHA.108.169722

47. Ahmadian M, Duncan RE, Sul HS. The skinny on fat: lipolysis and fatty acid utilization in adipocytes. Trends Endocrinol Metab (2009) 20:424-8. doi:10.1016/j.tem.2009.06.002

48. DeFronzo RA. Insulin resistance, lipotoxicity, type 2 diabetes and atherosclerosis: the missing links. The Claude Bernard Lecture 2009. Diabetologia (2010) 53:1270-87. doi:10.1007/s00125-010-1684-1

49. Mangubat M, Lutfy K, Lee ML, Pulido L, Stout D, Davis R, et al. Effect of nicotine on body composition in mice. JEndocrinol (2012) 212:317-26. doi:10.1530/JOE-11-0350

50. Sinha-Hikim I, Friedman TC, Shin C-S, Lee D, Ivey R, Sinha-Hikim AP. Nicotine in combination with a high-fat diet causes intramyocellular mitochondrial abnormalities in male mice. Endocrinology (2014) 155:865-72. doi:10.1210/en.2013-1795

51. Ahmadian M, Abbott MJ, Tang T, Hudak CSS, Kim Y, Bruss M, et al. Desnutrin/ ATGL is regulated by AMPK and is required for a brown adipose phenotype. Cell Metab (2011) 13:739-48. doi:10.1016/j.cmet.2011.05.002

52. Bozaykut P, Sahin A, Karademir B, Ozer NK. Endoplasmic reticulum stress related molecular mechanisms in nonalcoholic steatohepatitis. Mech Ageing Dev (2016) 157:17-29. doi:10.1016/j.mad.2016.07.001

53. Kammoun HL, Hainault I, Ferre P, Foufelle F. Nutritional related liver disease: targetting the endoplasmic reticulum stress. Curr Opin Clin Nutr Metab Care (2009) 12:575-82. doi:10.1097/MCO.0b013e32833189db

54. Motohashi H, Yamamoto M. Nrf2-Keap1 defines a physiologically important stress response mechanism. Trends Mol Med (2004) 10:549-57. doi:10.1016/ j.molmed.2004.09.003

55. Nguyen T, Nioi P, Pickett CB. The Nrf2-antioxidant response element signaling pathway and its activation by oxidative stress. J Biol Chem (2009) 284:13291-5. doi:10.1074/jbc.R900010200

56. Kensler TW, Wakabayashi N, Biswal S. Cell survival responses to environmental stress via the Keap1-Nrf2-ARE pathway. Ann Rev Pharmacol Toxicol (2007) 47:89-116. doi:10.1146/annurev.pharmtox.46.120604.141046

57. de Vries HE, Witte M, Hondius D, Rozemuller AJ, Drukarch B, Hoozemans J, et al. Nrf2-induced antioxidant protection: a promising target to counteract ROS-mediated damage in neurodegenerative disease? Free Radic Biol Med (2008) 45:1375-83. doi:10.1016/j.freeradbiomed.2008.09.001

58. Chowdhry S, Nazmy MH, Meakin PJ, Dinkova-Kostova AT, Walsh SV, Tsujita T, et al. Loss of Nrf2 markedly exacerbates nonalcoholic steatohepatitis. Free Radic Biol Med (2010) 48:357-71. doi:10.1016/j.freeradbiomed.2009. 11.007

59. Zhang YKJ, Yeager RL, Tanaka Y, Klaassen CD. Enhanced expression of Nrf2 in mice attenuates the fatty liver produced by a methionine- and choline-deficient diet. Toxicol Appl Pharmacol (2010) 245:326-34. doi:10.1016/j. taap.2010.03.016

60. Sinha-Hikim I, Sinha-Hikim AP, Shen R, Kim H, French SW, Vazari ND, et al. A novel cystine based antioxidant attenuates oxidative stress and hepatic steatosis in diet-induced obese mice. Exp Mol Pathol (2011) 91:419-28. doi:10.1016/j.yexmp.2011.04.009

61. Barreyro FJ, Kobayashi S, Bronk SF, Werneburg NW, Malhi H, Gores GJ. Transcriptional regulation of Bim by FoxO3A mediates hepatocyte lipoapoptosis. JBiol Chem (2007) 282:2741-54. doi:10.1074/jbc. M704391200
62. Cazanave SC, Mott JL, Elmi NA, Bronk SF, Werneburg NW, Akazawa Y, et al. JNK1-dependent PUMA expression contributes to hepatocyte lipoapoptosis. J Biol Chem (2009) 284:26591-602. doi:10.1074/jbc.M109.022491

63. Crowley-Weber CL, Dvorakova K, Crowley C, Bernstein H, Bernstein C, Garewal H, et al. Nicotine increases oxidative stress, activates NF-kappa B and GRP78, induces apoptosis and sensitizes cells to genotoxic/xenobiotic stresses by a multiple stress inducer, deoxycholate: relevance to colon carcinogenesis. Chem Biol Interact (2003) 145:53-66. doi:10.1016/S0009-2797(02)00162-X

64. Zabala V, Tong M, Yu R, Ramirez T, Yalcin EB, Balbo S, et al. Potebtial contribution of tobacco nicotine-derived nitrosamine ketone (NNK) in the pathogenesis of steatohepatitis in a chronic plus binge rat model of alcoholic liver disease. Alcohol Alcohol (2015) 50:118-31. doi:10.1093/alcalc/agu083

65. Li T, Feng R, Zhao C, Wang Y, Wang J, Liu S, et al. Dimethylarginine dimethylaminohydrolase 1 protects against high fat diet induced hepatic steatosis and insulin resistance in mice. Antioxid Redox Signal (2016). doi:10.1089/ ars.2016.6742

66. Mosinski JD, Pagadala MR, Mulya A, Huang H, Dan O, Shimizu H, et al. Gastric bypass surgery is protective from high-fat diet-induced non-alchoholic fatty liver disease and hepatic endoplasmic reticulum stress. Acta Physiol (Oxf) (2016) 217:141-51. doi:10.1111/apha.12640

67. Spencer MD, Hamp TJ, Reid RW, Fischer LM, Zeisel SH, Fodor AA. Association between composition of the human gastrointestinal microbiome and developmenet of fatty liver with choline deficiency. Gastroenterology (2011) 140:976-86. doi:10.1053/j.gastro.2010.11.049

68. Tremaroli V, Backhed F. Functional interactions between the gut microbiota and host metabolism. Nature (2012) 489:242-9. doi:10.1038/nature11552

69. Leung C, Rivera L, Firness JB, Angus PW. The role of gut microbiota in NAFLD. Nat Rev Gastroenterol Hepatol (2016) 13:412-25. doi:10.1038/ nrgastro.2016.85

70. Miele L, Valenza V, La Torre G, Montalto M, Cammarota G, Ricci R, et al. Increased intestinal permeability and tight junction alterations in nonalcoholic fatty liver disease. Hepatology (2009) 49:1877-87. doi:10.1002/hep.22848

71. Turnbaugh PJ, Ley RE, Mahowald MA, Magrini V, Mardis ER, Gordon JI. An obesity-associated gut microbiome with increased capacity for energy harvest. Nature (2006) 444:1027-31. doi:10.1038/nature05414

72. Den Besten G, Bleeker A, Gerding A, van Eunen K, Havinga R, van Dijk TH, et al. Short-chain fatty acids protect against high-fat diet-induced obesity via a PPARg-dependent switch from lipogenesis to fat oxidation. Diabetes (2015) 64:2398-408. doi:10.2337/db14-1213

73. Wang H, Zhao JX, Hu N, Ren J, Du M, Zhu MJ. Side-stream smoking reduces intestinal inflammation and increases expression of tight junction proteins. World J Gatroenterol (2012) 18:180-7. doi:10.3748/wjg.v18.i18.2180

74. Biedermann L, Zeitz J, Mwinyi J, Sutter-Minder E, Rehman A, Ott SJ, et al Smoking cessation induces profound changes in the composition if the intestinal microbiota in humans. PLose One (2013) 8:e59260. doi:10.1371/journal. pone. 0059260

Conflict of Interest Statement: The authors declare that the research was conducted in the absence of any commercial or financial relationships that could be construed as a potential conflict of interest.

Copyright $\odot 2017$ Sinha-Hikim, Sinha-Hikim and Friedman. This is an open-access article distributed under the terms of the Creative Commons Attribution License (CC $B Y)$. The use, distribution or reproduction in other forums is permitted, provided the original author(s) or licensor are credited and that the original publication in this journal is cited, in accordance with accepted academic practice. No use, distribution or reproduction is permitted which does not comply with these terms. 